\section{Fresh pathways to follow}

\section{Dani P. Bolognesi}

THE intricate mechanisms used by the immune system to recognize antigens are of great interest not only to immunologists but also to those involved in vaccine development. The trend over the past several years has been to group antigens into two categories according to the response they induce in the immune system (for review, see ref. 1). Nonreplicating entities that enter the antigenpresenting cell from outside (exogenously) are processed in the endosomal compartment and presented at the cell surface in association with class II major histocompatibility complex (MHC) molecules. This activates $\mathrm{CD}^{+}$helper T cells which, among other things, are required for the production of antibodies as well as $\mathrm{CD} 4^{+}$class II-restricted cytotoxic T lymphocytes (CTL). Alternatively, antigens enter a different processing pathway which is functional for proteins such as viral proteins that are synthesized inside the cell (endogenously). In this case, association is with MHC class I molecules and it is this complex that primes $\mathrm{CD} 8^{+}$ CTL. From the standpoint of immunization with anything other than an infectious agent, it has been something of a dilemma to design non-replicating immunogens that could allow processing and presentation by both class I and class II MHC. But several studies have indicated that these pathways are not as clear-cut as was once thought. For example on page 873 of this issue $^{2}$, Takahashi and colleagues now report that a unique subunit immunogen which induces neutralizing antibodies against human immunodeficiency virus (HIV) (B. Morein, personal communication) can also prime MHC class I-restricted HIV-specific CD8 ${ }^{+}$CTL.

Other examples are to be found in the generation of MHC class I-restricted CTL against soluble ovalbumin ${ }^{3}$ and against influenza virus peptides attached to 'lipid feet $^{\prime 4}$. On the other side of the coin is the novel observation that influenza virus proteins processed by the endogenous pathway can associate not only with $\mathrm{MHC}$ class I but also with class II molecules ${ }^{5}$. Association of endogenously synthesized antigens with class II MHC also occurs in the case of hepatitis B virus ${ }^{6}$ and for HIV envelope antigens synthesized inside the cell $^{7}$. In both of these examples, processing occurs in the endosomal compartment and is therefore distinct from the influenza case ${ }^{5}$. As investigation continues along these lines, models for antigen processing and presentation will probably converge further (see box), as already anticipated ${ }^{5.7 .8}$

These studies emphasize the need to understand better the different ways in which exogenous and endogenous antigens are processed and compartmentalized in cells. With regard to exogenous antigens, one might look to some mammalian viruses for clues. Viruses with membrane envelopes can penetrate cells by two mechanisms, one by fusion within endocytic vesicles, the other by direct fusion with the plasma cell membrane. It would be of interest to know how the appropriate entry route is selected. The answer may lie in the viral fusogenic proteins themselves and a more satisfactory definition of their interactions with the cell surface may help us to make cells respond selectively even to inert subunit immunogens. Manipulations with inactivated influenza virus preparations already look promising ${ }^{9}$. Influenza virus normally infects by fusion at low $\mathrm{pH}$ in endocytic vesicles, but heat inactivation of the virus causes fusion that allows the viral antigens to bypass the endosomal compartment and so to induce MHC class I-restricted CTL. Curiously, an opposite effect is also possible: for example, live measles virus fuses directly with the plasma membrane and yet preferentially induces a class IIrestricted CTL response ${ }^{10}$.

The precise mechanisms by which the influenza peptide derivatives ${ }^{4}$ and the immune-stimulating complexes (ISCOMs) of Takahashi et al. ${ }^{2}$ enter cells are not known. Both are associated with lipid, and it could be that the lipid is responsible not only for entry, but also for the susceptibility to processing by the endogenous pathway. On the other hand, as recognition by class II-restricted CTL of endogenously synthesized HIV envelope glycoproteins needs a membrane anchor, lipid/protein interactions must be important for delivery of endogenous antigens to the compartments where processing and association with MHC class II products can occur. One could speculate that antigens that remain associated with membranes (both within and outside the cell) may be destined for the class II pathway, whereas those that do not (but can still penetrate cells in the case of exogenous antigens) are channelled to the class $I$ pathway. This might be the case for viral core versus certain envelope antigens, for example. It could also be relevant to measles virus if the dominant CTL epitopes reside in components that associate with membranes.

For HIV vaccine researchers this is welcome news, principally because of the widely held belief that protective immune responses must be directed against both free virus and infected cells. Until now, only attenuated HIV preparations or replicating recombinant vectors were candidates, but the former are excluded because of safety considerations and the latter are still in the developmental stages. The prospect that subunit or peptide immunogens could be endowed with properties enabling them to enter either or both of the main processing pathways and stimulate a full range of immunity will receive considerable attention. One wonders too whether the protective

\section{Pathways of antigen processing} and presentation

\section{Exogenous antigens}

Endogenous antigens

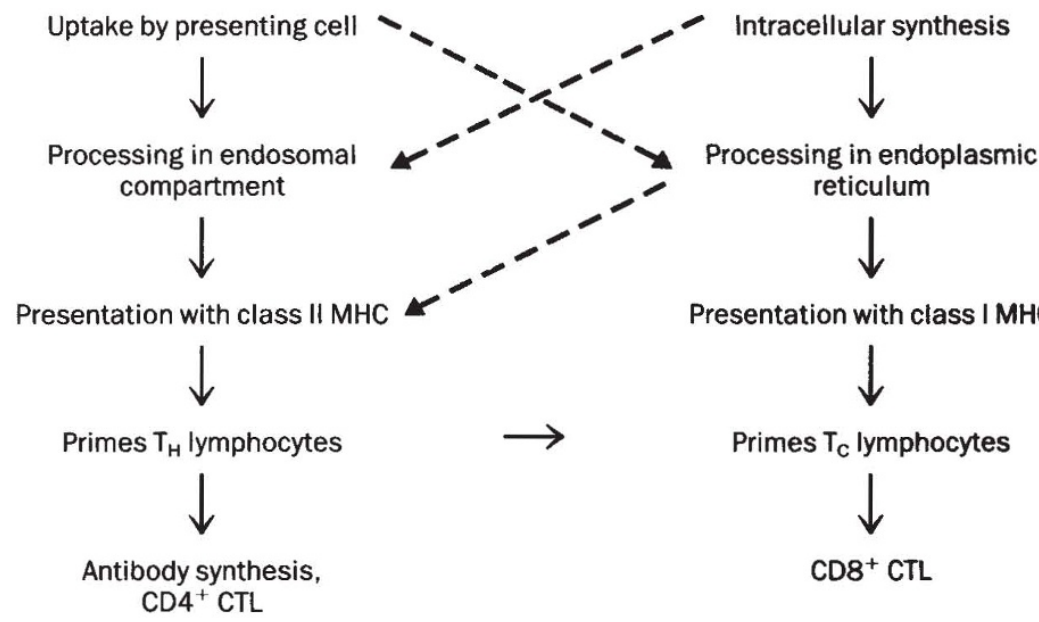

Solid arrows indicate current models and may represent primary pathways. Aiternative pathways (dashed arrows) have recently emerged, the prevalence and efficiency of which may depend on antigen formulation or the nature of the antigen itself. $T_{H}$, helper $T$ cell; $T_{C}$, cytotoxic T cell; CTL, cytotoxic T lymphocytes. 
response obtained with recent inactivated simian and human immunodeficiency viral vaccines ${ }^{11-13}$ could have included a CTL component, because the replicating forms of these viruses fuse directly with the plasma cell membrane ${ }^{14}$. But the use of killed HIV preparations for human vaccines carries considerable risk, and structures like the ISCOMs bearing only selected viral components may be attractive alternatives. In this regard the success of using ISCOMs to introduce whole viral proteins into cells for induction of CTL may be important, because it is likely that several T-cell epitopes will be needed both for priming and to overcome allotype restriction. Nevertheless, inclusion of key epitopes may be desirable and the target region described by Takahashi et al. ${ }^{1}$ is an important one because it is immunodominant for both neutralizing antibodies and CTL. Its drawback lies in its variability, although this might be overcome with appropriate cocktails ${ }^{15-17}$.

These results will stimulate a great deal of further research. But their ultimate value may depend on the extent to which TRANSCRIPTION INITIATION

\section{In search of the single factor}

\section{Marvin R. Paule}

EUKARYOTIC RNA polymerases are unable to recognize and transcribe from promoters at the beginning of genes without the aid of additional proteins, the general transcription factors. The three polymerases found in eukaryotic nuclei transcribe different sets of genes, and the number of factors associated with the initiation process increases as the variety of genes transcribed by the polymerase increases. Until now, the sole exception to the requirement for several transcription factors in eurkaryotes was that of ribosomal RNA transcription by polymerase I from Acanthamoeba, for which a single ancillary protein (TIF-I) is required ${ }^{1,2}$. But Kassavetis et al. $^{3}$ now report that only one of the three factors involved in transcription mediated by polymerase III in yeast, TFIIIB, is truly a transcription initiation factor. The others (TFIIIA and TFIIIC) are assembly factors responsible for loading the fundamental factor onto its site on the DNA. This observation means that the mechanisms described for rRNA transcription in Acanthamoeba, and now for transcription of yeast polymerase III genes, may be universal for eukaryotic transcription initiation - that is, that only one factor bound upstream of the transcription start site (dubbed +1 ) is needed to direct the polymerase to its binding site. The fundamental initiation factor can direct several rounds of initiation, and - most importantly - Kassavetis et al. show the addi- the alternative pathways can be used (see figure), particularly in vaccine development and immunotherapy.

Dani P. Bolognesi is in the Department of Surgery, and the Department of Microbiology and Immunology, Box 2926, Duke University Medical Center, Durham, North Carolina 27710, USA

1. Germain, R. Nature 322, 687-689 (1986)

2. Takahasi, H. et al. Nature $344,873-875$ (1990)

3. Staerz, U.D., Karasuyama, H. \& Garner, A.M. Nature 329. 449-451 (1987)

4. Deres, K. et al. Nature 342, 561-564 (1989).

5. Nuchtern, J.G., Biddison, W.E. \& Klausner, R.D. Nature 343, 74-76 (1990)

6. Polydefkis, M. et al. J. exp. Med. 171, 875-887 (1990).

7. Jin, Y. et al. J. exp. Med. 168, 293-306 (1988)

8. Long, E. O. \& Jacobson, S. J. Immun. 10, 45-48 (1989).

9. Yewdell, J.W., Bennink, J.R. \& Hosaka, Y. Science 239, $637-639(1988)$

10. Sekaly, R.P. et al. Proc natn. Acad Sci. US.A.85, 1209-1212 (1988)

11. Derosiers, R.C. et al. Proc. natn. Acad. Sci. U.S.A. 86 6353-6357 (1989)

12. Murphy-Corb, M. et al. Science 246, 1293-1297 (1989).

. Gibbs, C.J. et al. Vth Int. Conf. on AIDS, Montreal, Canada (Abstr. C. Th. C.0. 46, 1989)

14. Stein, B.S. et al. Cel/ 49, 659-668 (1987)

15. Putney, S.D. et al. Vth International conference on AIDS, Montreal, Canada (Abstr. W.C.O. 18, 1989).

16. Zwart, G. et al. Lancet II, 474 (1990).

17. Devash, Y. et al. AlDS Res. hum. Retrovir. (in the press)
They stripped TFIIIA and TFIIIC from the templates by using high salt concentration or heparin, and then isolated the DNA-protein complexes by sizeexclusion chromatography. Only TFIIIB complexes remained, and surprisingly, they retained the capacity for several rounds of transcription. The factors that had been stripped from the template were able to assemble fresh TFIIIB on a new template; TFIIIA and TFIIIC are therefore assembly factors, and are not required for transcription initiation per se.

Pre-initiation complexes of TRNA and 5S RNA transcription in yeast ${ }^{3.5 .10}$ and rRNA transcription in Acanthamoeba ${ }^{2,11,12}$ have been 'visualized' using footprinting techniques. Yeast TFIIIB was found to protect the DNA template between about 10 and 40 base pairs upstream of +1 on $5 \mathrm{~S}$ RNA and tRNA genes. The single Acanthamoeba rRNA transcription initiation factor (TIF-I) protected between about -12 and -70 base pairs, forming a stable complex which remained bound through several rounds of transcription (see figure). The two factors therefore form similar complexes upstream of the transcription start site.

The first footprints of a eukaryotic polymerase on a promoter demonstrated that Acanthamoeba RNA polymerase binds just downstream of TIF-I, protecting over $34 \mathrm{bp}$, to +18 , from DNase I digestion ${ }^{2}$. Replacement of the protected region with a variety of bacterial sequences showed that there are no DNA sequence-dependent contacts made by polymerase, but instead, the enzyme is positioned on the promoter by proteinprotein contacts with TIF-I ${ }^{13}$. Similarly, polymerase III protects 23 bp (for $5 \mathrm{~S}$ RNA genes) or 28 bp (for tRNA genes) of DNA just downstream of TFIIIB. Earlier studies by Sakonju et ll $^{1+}$ suggest that the binding of polymerase to $5 \mathrm{~S}$ RNA genes is also sequence-independent. To prove that the extended footprints are due to protection by polymerase and not to a conformational change in the previously bound factor, the polymerase I (refs 11, 13) and III (ref. 2) systems were supplied with a nucleotide mixture that allows the polymerase to make only a short RNA product. Addition of a mixture lacking GTP resulted in the polymerase stalling part way down the template. As predicted, the putative polymerase footprints moved part way down the DNA. Addition of all four nucleoside triphosphates resulted in total disappearance of the polymerase footprints.

Significantly, the Acanthamoeba TIF-I footprint remains unaltered during initiation, showing that TIF-I remains bound through several rounds of transcription $^{11,12}$. In the polymerase III systems, the TIIIB footprint was also unchanged after partial translocation of the polymerase down the template. Furthermore, 